\title{
That word doesn't exist in my language: teaching and learning Laban/Bartenieff Movement System in multiple languages
}

\section{缺失的语言 : 在多语中讲授学习拉班/芭特妮芙动作系统}

\author{
Alexandra Baybutt ${ }^{1}$, Joanna Brotman², Stephanie de \\ Bruijckere $^{3}$, Catherine Maguire ${ }^{4}$, Karen Studd ${ }^{4}$ \\ ${ }^{1}$ Middlesex University, UK \\ ${ }^{2}$ The Dalton School in New York City, US \\ ${ }^{3}$ Hogeschool Zuid Sittard, The Netherlands \\ ${ }^{4}$ Whole Movement and the Laban/Bartenieff Institute of \\ Movement Studies, US
}

\begin{abstract}
This article shares insights from teachers of the Laban/Bartenieff Movement System (LBMS) dealing with translation in different ways in the classroom. We problematize the notion of translation and consider the challenges and values of our attempts to translate that produce significant spaces of learning. From these, we elaborate upon reflexive models of teaching and learning, fundamental to LBMS. We use our experiences to highlight the role of Motif within LBMS and propose curriculum modifications and additions.
\end{abstract}

Keywords: Translation, Teaching, Laban, Bartenieff, Movement

\section{摘要}

这篇文章分享了讲授拉班/芭特妮芙动作系统 (LBMS) 时将教学内容翻译成不同语言时 的领悟。我们对 “翻译” 的传统概念提出疑问，并探讨翻译时所面临的挑战以及努力的 价值。这个翻译的过程创建了重要的学习空间，因为它给了教学反馈和互动的机会，这 是学习LBMS的基础。我们利用这个经历强调了主题舞谱在LBMS中的角色，并对教学 提出了修改和增加的建议。

关键词: 翻译，教学，拉班，芭特妮芙，动作

This article shares insights from teachers/facilitators of the Laban/Bartenieff Movement System (LBMS). The Laban/Bartenieff Movement System is a comprehensive system used in understanding multiple aspects of the patterns of human movement. LBMS methodology incorporates a theoretical framework and clearly delineated language for describing movement. This article focuses on what we have learned in recent years about processes of learning, and about how a body of knowledge is enriched through the dynamic of teaching and learning across many languages. We share and debate our experiences from a variety of linguistic cultures, highlighting the rationales for our 
tactics and experiments that ground specific differences. These may be read as suggestions - not necessarily as guidelines - for future consideration. By closely exploring and attending to context-specific needs where no one size-fits-all, the process-oriented perspective we have for learning and growth leads us to argue that each specific context reveals the information for how to respond and facilitate learning. What remains constant is an educational commitment to disseminating the tools and systems of the work of Rudolf Laban and Irmgard Bartenieff. We therefore value ongoing training and reflection of our observation skills that includes attention to the words we use - or don't use - in embodying concepts and movement facilitation. In the spirit of LBMS as an ongoing process of learning for us as educators, various concepts from the system itself are used to further contextualize the debates - modelling the creative ways in which this system allows for new connections and meaning to be made.

Alexandra Baybutt, Stephanie de Bruijckere, Joanna Brotman and Cat Maguire all have worked with Karen Studd who has coordinated and led modular certification programs in LBMS, accredited by LIMS in New York, USA, since 2006. These modular programs have taken place in Belgium, Canada, China, Israel, Mexico, Scotland and USA. This article is co-authored in the spirit of the community learning model underpinning the teaching and learning of LBMS.

\section{Codification and distillation into shared representations}

\section{Thematic Dualities}

Fundamental patterns of duality are a key aspect of the Laban Bartenieff Movement Systems' capacity to contain wholeness. They operate as a whole - for example Inner/Outer - and are used in that way in a shifting play of foreground/background that defines work of LBMS. These Thematic Dualities are models and representations of relationships - not the thing in itself. Unique individuality is explored through LBMS by a duality of Individual/Universal. The individual experience, or the personal, is not only framed but analyzed and synthesized alongside 'universal'. When we remove either part (Universal or Individual) from the whole (Individual/Universal), synthesis is not possible and we are no longer working within the system. This duality is particularly useful in teaching LBMS to see our personal patterns against a universal norm in which 'universal' becomes provisional rather than absolute when cultural 'norms' appear also as variable.

LBMS encompasses both the individual and universal nature of movement. This is an ambitious claim without further explication. In LBMS, 'universal' operates in a similar way to that some geometrical absolutes are used. For example, the pure Diagonals and the pure Dimensional pulls are clear spatial examples that are never really there but visualized/imagined, but we can closely approximate them in/through movement. That our body exists as weight in gravity with a base of support is another shared, universal aspect through which movement is understood. It is critical for effective use of LBMS that a concept and its meaning only exists within a context. This ensures we avoid the pitfalls of one-sided analysis - which precludes synthesis. The fluid interplay of Thematic Dualities - especially Individual/Universal - is opened up even more by multilingual and multicultural learning communities. The constant shift of what is 
foregrounded and backgrounded is what allows the system to hold diversity and commonality simultaneously.

\section{Individuality, culture, context}

Each individual's experience of themselves in the world is unique. We are aware of our own awareness such that no other could enter fully into that uniqueness, so each other is already a different being with individual ontology. I shall retain Descartes's insistence on discrete consciousness as the 'ontological essence of being human' (Nigel Rapport, 2014: 332). In our individuality, we are always situated (as a body) in a certain time and space. Taking individuality and being situated together means that even if we ostensibly 'share' the same first language, differences in perception and deployment of concepts (both individually and culturally determined) nevertheless lend nuanced subtle variations or stark differences in how we move through life. We don't necessarily share an understanding of a concept even if we do speak the 'same' language because our associations are unique. Observing comprehension and perception from this perspective of 'shared language' already presupposes a mono-linguistic normative paradigm that ignores those individuals who grow up in bi- or even tri-lingual families and contexts.

Let's also start from acknowledging language is always an approximation, unfinished, the result of an embodied experience in relationship (with human/non-human agents) in the world. Following anthropologists Thomas Csordas (1994) and Marilyn Strathern (1996), culture does not just inform embodied experience; embodied experience is itself culturally constituted (Lawrence Shapiro, 2011). As anthropologist Anthony Wallace points out, 'cultures do exist and societies do survive' (Wallace, 1961: 131). And lastly, that translation from one language to another is impossible: something slips out, other things are added. It is interesting that neuroscientists can track and trace where in the brain such activity appears; though in the studio and faculty meetings, we must navigate the transformation of concepts produced through translation through practical action. LBMS is a particularly rich frame through which to explore individuality and the cultural specificities we bring into the room - as well as those generated in it. Even though we work in studios generally with little furniture, it's not 'empty'; the context is always specific and can change, or be changed, through our narratives and analogies.

\section{Laban's legacies, many narratives}

LBMS, as a branch of the work of Rudolf Laban, is part of his wide-reaching legacy since the 1950s onward. 'Laban' exists as a 'global' idea, appearing 'internationally' in many professional, artistic and educational contexts. Such terms 'global' and 'international' can flatten specificity; each student is meeting it in a particular time and place, so what are the experiences in that encounter and how are they shaped? These are happening in a flow of time - not in a fixed concept of the 'international'. In the multiplicity of approaches to Laban's work, as well as the lack of consensus in these many branches over language, terms, concepts and so forth, are arguably a necessary part of its vitality. Languages that do not change or are not used tend to disappear or, at least, fade (Latin; Esperanto). Bodies of knowledge that are not found to have value or relevance, fade. 
The increase of markets for 'health and wellness', or of community dance practices for example, are traceable to the movement researchers and pioneers of the start of the $20^{\text {th }}$ century in Europe and North America - of which Laban can be included. Taking a Foucauldian model of genealogy that eschews confident points of origin and instead appreciates a more gradual and messy process of accumulation and sedimentation (Franklin, 2014: 14; Foucault, 1984: 58-59), the point is less to split hairs on provenance than to work with this multiplicity of discourses and applications of movement observation and analysis, and to question so-called 'norms'. Although as practitioners of LBMS we value, or rather, require, consensus in order for the system to function as a useful discourse and modality in our work, finding consensus need not negate the frequent dissensus found in our individual experiences and perceptions.

The concrete limitations of language afford insight into the complexity of movement. Language often serves as a privileged model of our experience to paraphrase theorist of economics Dani Rodrik (2015) however, models are never true - but there is truth in models. We can understand the 'real' phenomenon only by simplifying it, hence the provisional nature of language remains at the forefront of this discussion. The subtleties of language open onto the subtleties of movement and vice versa. Whilst language issues can become a problem in the negative sense, it is more generative to use 'problem' in the philosophical sense as a notion of opening possibilities (following dramaturg Bojana Cvejić's Choreographing Problems, 2016). In this way, the problems of language are tied to the clarifying nature of language. Much like there are no fixed points in space, there are no concrete terms; we temporarily stabilize a concept in order to illuminate something (ie. a system for movement analysis such as LBMS), but already that sets the term off on a rhizome of associations and interconnections (how LBMS connects to other somatic modalities, therapeutic interventions, more established academic disciplines and so on and so forth). The point to make is that in the experience of debating semantics is the site of learning that LBMS opens discursively - and recursively - in pursuing an iterative process of movement embodiment and observation.

\section{Languages as politically embedded and embodied}

The specific LBMS language, tools and concepts work to encapsulate a particular facet for the clarification and consensus of observable movement and, to an extent, the experience of it. In training observers, we require increased embodiment of a range of subtleties in order to perceive and articulate the differences and to be able to enter into a wide range of possibilities in movement. We aim to facilitate the learning of these tools - not as dogma or reduction but as process and self-discovery. Learning how to use LBMS is creative and a life-long study; hence a debate on the flexibility and rigidity of language is one such way to explore this body of knowledge. In the studio, we find ourselves and our students sometimes using LBMS words interchangeably, but they are not the same thing. The words (and symbols: the system of Motif) can allow for both specificity and flexibility - which we consider particularly valuable for creating a bridge for communication - but also a boundary with some 'fixed points' in a co-created space.

'Mobility/Stability' - one of the Thematic Dualities of LBMS - immediately springs to mind in considering questions of malleability and fixity. Language, or to be more 
specific, words, can be considered containers for experience and words, like movement, reflect the choice(s) of the mover/speaker. These choices reflect their values and include habits related to background and culture. And as is true for all containers, they can serve to hold and support but can isolate and separate; it is this simultaneity of their formulation and use that is particularly dynamic. Different kinds of containers have different kinds of shapes and edges - for example, those that can/cannot adapt to the content (like a wine-skin vs a wine bottle) - or that have permeable boundaries (like a woven basket or fishing net). And indeed, decisions needed to be made whether we as a group of writers contributing to this article choose to adopt the British or American English spellings. Deciding 'house style' for journals and authorship is a practical one with geopolitical resonance.

Our experiences emerge from using spoken and written language and embodiment to facilitate LBMS; this is two-fold, hinted at in the term 'facilitate'. Firstly, as stated, there are specific terms to teach - ontologically bound concepts - the 'universal' aspects of which precede cultural specificity or at least attempt to hold them in abeyance in order to be re-situated. Second, there are embodied experiences to elicit from the students. For example, specific qualities of moving might already be available for as individual and therefore not necessarily needing to be 'taught' as entirely 'new' concepts, but instead brought to conscious awareness of their subtly and distinctness through moving itself. Here the image of the moebius strip becomes particularly pertinent to emphasize this dynamic of how information from within and without resonates in a given context of learning with others. The facilitative process is interactive, and evocative other Thematic Dualities of LBMS - Inner/Outer and Function/Expression. There is more to be said that is beyond the scope of this article concerning facilitating LBMS with those with special needs, disabilities and a larger range of neurodiversity that what we have encountered on the modular programs.

\section{Learning and using Motif symbols}

Irrespective of the (dis)advantages of 'shared' language or contexts, comprehension still needs support through different tools. The significance of Motif writing - a tool used in teaching and learning LBMS - comes in here. Motif is a symbolic representation of movement and not the movement itself (a form of iconic sign, Eco, 1984: 16). The nature of Motif, by its design, shares aspects of both the verbal and non-verbal realms we value in movement analysis training - thus it can be a valuable tool in the process. Motif can be a bridge between movement expression/experience and the analytical process of describing/identifying movement patterns. This allows Motif to be a bridge between the analysis and synthesis ends of LBMS. Motif as a language attempts to avoid some of the pitfalls of linguistic verbal written languages. Though Motif can also serve as a tool for recording certain essences of movement, it is not only for such processes of creating an archive or document. More akin to sketching or note-taking, Motif can both reveal and represent movement patterns and can be facilitated through iterative partner observation tasks, for example. It can also be useful in challenging patterns (the process of re-patterning or expanding range). Though related to Labanotation, Motif is not a de facto branch of it, nor is it a shorthand version. Labanotation has a different historical 
development and different intent than Motif. Motif is a shorthand of the language of LBMS and is succinct - not only in the reduction of how much one has to say out loud. The Shape Quality and Effort symbols work in a modular format in which the choice of symbol can communicate, for example, three qualities of movement happening simultaneously. To say these aloud implies their succession rather than their simultaneity. In the studio, we and our students go from moving and talking to grabbing a piece of paper and scribbling some symbols then back to moving, making this process as fluid as possible rather than somehow separate, like splitting writing from reading or speaking from listening. The use of Motif, therefore, deployed in different ways as part of processes, overcomes several of the challenges not only of multi-language learning spaces but also of facilitating movement embodiment.

\section{Specific examples of approaching translation in teaching and learning}

Laban wrote originally in German and then in English. A note is needed on the rather hegemonic English-language dominance in teaching and learning in arts, therapies and humanities and other professional practices around the world. This can lead to a level of complacency around bodies of knowledge as being stable and authoritative. To avoid or at least acknowledge such ethnocentrism, it can help to increase the specificity of choices and make explicit reference to relativism. For example, the use of terms from Laban in English show variants across different eras and geographical locations. In the UK, for example, do you use Sudden or Quick, Flexible or Indirect, Remote State or Near State? It will depend on who your teachers were, which texts you read, which texts were available to you and when. Practitioners of Laban might share the same concept but have a different name for it. This evokes Emily Beausoleil's articulation of responsibility being response-ability (2015) as a dispositional ethics of encountering difference shaped by affectivity. What does this mean in practice?

When Stephanie de Bruijckere teaches LBMS at AgapeBelgium with Belgian students, she uses English words - choosing not to translate into Dutch because, for her, it's another rhythm and dynamic. By contrast, when Sabine Fichter taught a module at AgapeBelgium, she used German words; de Bruijckere observed a difference in the movement of the students using German terms compared with those using English terms. In AgapeBelgium, though, there is no official 'translator' - these processes of clarification between languages happen less formally with students talking amongst themselves. People who speak different languages work together and help each other during the process of learning LBMS as a new language. By contrast (geographically) when Karen Studd was teaching in Montreal, she found that there were students who wanted terms translated into Quebecois; this created a discussion of specificity. Two Croatian students taking part in the CMA training in Scotland were regularly translating from the (then) Serbo-Croatian terms learnt at the Ana Maletic School in Zagreb in the 1970s into English. There are students who speak several languages who provide an additional layer of translation in the process. This interactive aspect between the students themselves is part of the necessary dynamics of learning. 
de Bruijckere learned LBMS as a student in New York, learning in English as a Dutch speaker. Even as a student, she noticed how her use of observation became crucial in grasping a concept, embodying it as a way to articulate it, all the while in the process of learning which is fraught with non-comprehension and vulnerability as well as with pleasure. In addition to how intensive observation supported her in the position of learner, de Bruijckere currently finds that, when she teaches in English, there is a preparation phrase that is needed to give her more time as she senses into what she is going to say. As a facilitator, acute observation practice as a learner, kinaesthetic attunement from artistic and therapeutic training and the experience of expanded time as duration needed in preparation for speaking all give her an opportunity to intuit the experiences of the students in that moment. She can relate to how they appear as she gives herself time to see their reactions. Impulsive phrasing in her speech is not possible but neither is it necessarily wished for; such speed would erase the gap she finds powerful for supporting learning.

From the perspectives of the facilitators operating as a temporary faculty in the modular programs designed in intensive formats (four classes per day - generally over consecutive days), LBMS is a knowledge practice that stands alone. Paradoxically, however, it intersects according to context with many other knowledge practices (the individuals in the room who are generally adult learners from a range of backgrounds and expertise and come in with different orders of knowledge). How we teach can become unmoored as failures to connect or communicate a concept provoke the experimentation of new tools. In this regard, the use of images, metaphors and analogies becomes - as de Bruijckere points out - important as she experiences students grasping a concept through an image. Learning a concept through movement of the body is also crucial and calls up the significance in LBMS of using Motif symbols to bridge between movement and words. de Bruijckere notes even the movement in the symbols themselves as impetus for embodiment.

Teaching LBMS in China in English involves working closely with a translator. The process of increased time for observing students' learning is reiterated by Karen Studd in gaining the opportunity to witness what could be absorbed by them. Rather than 'slowing down' as an impediment to an idealized smooth flow of learning over time, slowing down and re-phrasing the rhythms of speech units was found to be beneficial for teaching differently because of what is gained through a new perspective. As well as bringing material to learn in this encounter, the responsibility of the movement educator is partly to be in response to the flow of the room and its different needs and tempos. The attunement to each other is, at times, taken for granted as much of it is non-verbal; however these examples from working with a translator makes these processes more apparent for the students as well as the non-Mandarin speaking tutors.

Whilst acknowledging differences between practitioners in the Laban community as a larger notion, LBMS as a language gradually becomes shared as a context (Duranti and Goodwin, 1992: 7) over the course of a modular program. In the context of teaching in China in English with translation into Mandarin, once specific terms are translated and grasped conceptually by the students, the LBMS terms are then no longer translated each time but are spoken in their English form inside of all other translation so that the 
concepts are attached to their sound and to the LBMS language itself (as embodied by the facilitator also). This shows a collaborative approach to incorporating new language for concepts developed from an Anglo-centric frame in the flow of movement and concept learning that both transforms and retains specificity at the same time.

The opportunity of working with a translator so sensitive to LBMS and group processes has facilitated our learning, self-reflection and re-patterning. Katee Shen '沈 妍 is a translator in China who, over several years, has been working with several teachers of movement and arts therapy and of LBMS. Speaking with her, Baybutt, Studd and Maguire all could learn more about their own patterns of phrasing by hearing the differences Shen perceived from translating for them. Each of us have different styles and variations in how we shape simplicity and complexity. Phrasing is key in working with a translator and with the interaction rhythm of communication. Studd has found that 'less is more' and lets 'the movement be the conduit for creating meaning' something essential to keep in mind as a faculty in conveying ideas. She needed to modify her preference for long overlapping phrases (Complexity) and Quick Time when working with a translator. Initially this felt like 'dumbing down,' but she has since found more clarity around this pattern.

Maguire, working with the same translator, found a different phrasing pattern and experience. Her preference for discrete, impulsive phrases necessitated a 'dance' with the translator of pausing to allow the concepts to be fully explained in Mandarin. Like Studd, she discovered that succinct, imagistic language combined with explicative movement facilitated a smoother process of imparting information. Whilst some metaphors and references are very culturally specific, others have the capacity to be shared. When they aren't shared, it is a useful opening for a discussion of analogy and metaphor. 'There is a metaphor every time something unexplainable happens which the users of a language perceive as a metaphor' (Eco, 1984: 88).

This brings to mind another Thematic Duality of 'Exertion/Recuperation'. There is an additional exertion for the action of translation for the student in trying to absorb through a foreign language on top of the newness of the LBMS terminology. But this is also the case for the faculty in finding the interactive phrasing with the translator on top of the phrasing they are already invested in relation to conveying the curricular content and in, for example, providing a supportive ongoing monologue in leading an ongoing improvisational exploration or in cueing students at crucial times in a sequence.

Studd reflects upon how this process requires a letting go of a sense of control over how the facilitation occurs - and how mutual observation is occurring - in order to recognize that these experiences embody the community learning model for which she advocates. There is a full, rich learning potential in seeing two people teach, of one teacher seeing the interaction between the students and the other teacher/assistant, of seeing the translator teaching the faculty and so on. The interwoven synergy of co-learning makes visible how we learn to learn. If the translator is very skillful, they can pick these nuances up and this becomes the more-than of words where kinaesthetic empathy to oneself and the process is key. Building good working relations takes time, trial and error, discussion and generous communication. These examples attest to the variety of expression in teaching and learning. As de Bruijckere points out, there is no 
ultimate right way of facilitating because of the unknowable variety of ways of eliciting movement in the mover. As much as the words are useful, she is attentive to the movement of her students and clients - considering full expression to be all of it, not only verbal or kinaesthetic 'competency'.

\section{Insights for how we teach and what we teach}

The examples above demonstrate aspects of the learning community model underpinning the formats of the modular programs led by Studd. It is based on synergy produced from emergent process of creating - and of growth and development - that are found in all systems including language(s). These reflections lead us to consider tactics pertinent to designing and refining intensive modular learning formats, but also to questions of the curriculum content. Though the category of Shape within LBMS typically is articulated as comprising the majority of tools for engaging in and understanding relationality, we would argue that missing from the curriculum at present is a part that really addresses interaction. Despite working in interaction, interaction itself is not a formal part of the theory and technique, but it is an important part of the process. The self-discovery, growth and learning that takes place for students on the CMA trainings is profound, individual experience is not to be reduced, however we could become more explicit about interaction aspects.

What became apparent in this discussion together was the increased awareness on the link between movement, language, voice and breath. This continuum of Inner/Outer of breath-sound-word/song is movement, and, though their differentiations can be useful, they also prevent against more embodied, integrative explorations. Reasons for this are beyond the scope of this article but one brief thought is that this might connect to the disciplinary differences in teaching dance (students do not speak or vocalize) that historically impinge upon teaching styles.

However, we also can share our awareness of the use of our voices in teaching; for example the use of Time Effort, in how to guide people into inner sensing. There comes a moment when students are not necessarily taking in the content as words, but the texture/timbre. The performativity of facilitation and its affective intensities in the learning environment are as important to a theatre actor's narrative arc.

The emphasis on processes and experiences this article has tried to capture attests to the discoveries of languaging as learning. Bringing to consciousness and to articulation are profound processes through which the actual learning takes place. Languaging is embodied; LBMS practitioners perceive body as basis for knowing the world and this is the conduit through which learning happens. For facilitators of LBMS, as well as those newer to this work, we hope this article supports an appreciation of the dynamic between the language of it, the challenges of translation, and how to navigate working in multilanguage contexts sensitively.

\section{Acknowledgement}

This article emerged from a panel discussion held at the LIMS $40^{\text {th }}$ Anniversary event in 2018 in New York, USA, convened by Alexandra Baybutt. 


\section{About the Authors}

Alexandra Baybutt, artist, movement educator, Certified Movement Analyst (Laban/ Bartenieff Institute of Movement Studies), BA Laban, UK, MA Goldsmiths College, $\mathrm{UK}$, and currently $\mathrm{PhD}$ candidate at Middlesex University, UK, researching festival curation of contemporary performance in the former Yugoslav space.

Joanna Brotman, CMA, RSME (Registered Somatic Movement Educator), RSMT (Registered Somatic Movement Therapist), CET, is on the faculty of the LIMS Certification Programs in Israel and Amsterdam. Joanna is a professor in the Dance Education MA Program at Rutgers University and Chair of the Dance Department at The Dalton School in New York City. She is a contributor to PAJ: A Journal of Performance and Art (MIT Press).

Stephanie de Bruijckere, CMA, DMT (Hogeschool Zuid Sittard), Dance and Movement Therapist, teacher and coordinator of the Dance \& Movement Therapy and LBMS programmes atAgapeBelgium. Stephanieco-organizes AgapeBelgium with Niek Ghekiere. Catherine Maguire is a movement educator, dance artist, CMA,RSME, and teacher of the Laban/Bartenieff Movement System (LBMS). Cat has taught and co-coordinated CMA Programs in the US, Europe, Mexico and China. Cat is a consultant for the Robotics, Automation and Dance (RAD) Lab at the University of Illinois Urbana-Champaign.

Karen A. Studd, MA, Dance, University of Oregon, CMA(LIMS), RSMT, RSME. She is a Movement Analysis Training Program Coordinator and has taught LBMS in the US, Canada, Europe, Mexico, Israel and in China.Karen has also taught for the Dance Departments of the University of Oregon, the University of Wisconsin, George Washington University and George Mason University, where she was a tenured faculty for over twenty years.

\section{Conflicts of Interest}

The authors declare no conflict of interest.

\section{References}

Beausoleil, E. (2015). Embodying an Ethics of Response-ability. Borderlands, 14(2).

Cvejić, B. (2015). Choreographing Problems: Expressive Concepts in Contemporary Dance and Performance. Basingstoke, UK: Palgrave Macmillan.

Duranti, A. \& Goodwin, C. (1992). Language as an Interactive Phenomenon. Cambridge, UK: Cambridge University Press.

Eco, U. (1984). Semiotics and the Philosophy of Language. Bloomington, USA: Indiana University Press.

Foucault, M. (1972). Archeology of Knowledge and the Discourse on Language. New York, USA: Pantheon Books.

Franklin, S. (2014). Analogic Return: The Reproductive Life of Conceptuality. Theory, Culture \& Society, 31(2-3), 243-261.

Rapport, N. (2014). Social and Cultural Anthropology: The Key Concepts. Abingdon, UK: Routledge. Rodrik, D. (2015). Economic Rules. London, UK, New York, USA: W. W. Norton and Company. Lawrence S. (2011). Embodied Cognition. Abingdon, UK: Routledge. 